Robert Ott*, Swen Großmann, Alper Öner, Hüseyin Ince, Michael Stiehm, Niels Grabow, Klaus-Peter Schmitz and Stefan Siewert

\title{
Transcatheter mitral valve repair devices - in vitro studies on the influence of device-width on mitral regurgitation
}

\begin{abstract}
Mitral regurgitation (MR) is the most prevalent valvulopathy in the USA and the second most prevalent valvulopathy in Europe. Despite excellent clinical results of surgical mitral valve repair (SMVR), transcatheter-based mitral valve repair (MVR) procedures emerged as a feasible treatment option for surgically inoperable or high-risk patients suffering from clinically relevant MR. The current study investigates the impact of device-induced coaptationwidth on the hydrodynamic performance of insufficient mitral valves (MV) during left ventricular (LV) systole.

A non-calcified, pathological MV model (MVM) featuring a D-shaped MV annulus with an area of $7.6 \mathrm{~cm}^{2}$ and a flail gap in the A2-P2 region was employed in an experimental setup. Pressure gradient-volumetric flow rate $(\Delta p-Q)$ relations were investigated for steady-state backward flow with transvalvular pressure gradients ranging from $(0.75 \leq \Delta p \leq 177.36) \mathrm{mmHg}$. Glycerol-water mixture $\left(36 \%(\mathrm{v} / \mathrm{v})\right.$ glycerol in water) at $37^{\circ} \mathrm{C}$ with a density of $(1098.2 \pm 1.3) \mathrm{kg} \cdot \mathrm{m}^{-3}$ and a dynamic viscosity of $3.5 \mathrm{mPa} \cdot \mathrm{s}$ was used as circulatory fluid.

In order to determine the impact of the width of transcatheter MVR devices during LV-systole $\Delta p-Q$ relations were investigated for three MVM-configurations: (i) MVM without MVR device, (ii) MVM with one MVR device and (iii) MVM with two MVR devices implanted in the A2-P2 region. The MVR devices were manufactured from steel sheets with a thickness of $0.2 \mathrm{~mm}$ and feature arm lengths of $9.0 \mathrm{~mm}$ and a width of $5.0 \mathrm{~mm}$.
\end{abstract}

\footnotetext{
*Corresponding author: Robert Ott: Institute for ImplantTechnology and Biomaterials e.V., Friedrich-Barnewitz-Str. 4, Rostock, Germany, e-mail: robert.ott@uni-rostock.de Sebastian Kaule, Swen Großmann, Michael Stiehm, Klaus-Peter Schmitz, Stefan Siewert: Institute for ImplantTechnology and Biomaterials e.V., Rostock, Germany Alper Öner, Hüseyin Ince: Heart Center/Department of Cardiology, Rostock University Medical Center, Rostock, Germany

Klaus-Peter Schmitz, Niels Grabow: Institute for Biomedical Engineering, Rostock University Medical Center, Rostock, Germany
}

The conducted investigations show that the implantation of MVR devices in the A2-P2 region prevents the manifestation of an A2-P2 flail gap and thereby effectively reduces the retrograde blood flow during the LV-systole by $13 \%$ with one MVR device and $27 \%$ with two MVR devices implanted.

Thus, the application of two MVR devices with a combined device-induced width of $10 \mathrm{~mm}$ results in a better MR reduction than the implantation of one MVR device with a device-induced width of $5 \mathrm{~mm}$.

Keywords: Mitral Regurgitation, Mitral Valve Repair, MitraClip-System, Pascal-System, in vitro Model

https://doi.org/10.1515/cdbme-2020-3055

\section{Introduction}

The bicuspid mitral valve (MV) is a highly intricate anatomical structure located between the left atrium (LA) and the left ventricle (LV). The functional anatomy of the MV comprises four structures: (i) anterior and posterior leaflets, (ii) papillary muscles, (iii) chordae tendineae and (IV) mitral annulus [1]. Any intrinsic abnormality of the MV apparatus or disturbance of the aforementioned structures can lead to mitral regurgitation (MR) and thereby to systolic retrograde blood flow inducing a decrease of both LV-pressure and forward flow of oxygenated blood [1-3]. In terms of prevalence, MR is the most common valvulopathy in patients aged 75 years and older and the second most prevalent valvulopathy in Europe $[4,5]$.

After the introduction of surgical MV repair (SMVR) procedures, such as the edge-to-edge technique in 1991, SMVR evolved as the gold standard for the treatment of clinically relevant MR $[6,7]$. Despite the excellent clinical outcome of SMVR, transcatheter-based MVR devices, mimicking the surgical edge-to-edge technique, emerged as feasible treatment options for surgically inoperable or highrisk patients suffering from clinically relevant $\mathrm{MR}[8,9]$. 
Although clinical data regarding the performance of transcatheter-based MVR devices are broadly available, the impact of device-induced coaptation-width and -area on the hydrodynamic performance of the MV has not been fully understood yet. To address this problem, we investigated the hydrodynamic performance of a pathological MV model (MVM) after implanting one and two custom-made MVR devices with an arm length of $9 \mathrm{~mm}$ and a width of $5 \mathrm{~mm}$ in the A2-P2 region, respectively.

\section{Materials and methods}

\subsection{Experimental setup and circulatory fluid}

For in vitro investigations an experimental setup as described in a previous work was used [10]. The $\Delta p-Q$ relations were investigated for steady-state backward flow applying physiological transvalvular pressure gradients ranging from $(0.75 \leq \Delta p \leq 177.36) \mathrm{mmHg}$. As suggested by Lim et al. a glycerol-water mixture $(36 \%(\mathrm{v} / \mathrm{v})$ glycerol in water) at $37^{\circ} \mathrm{C}$ was utilized as a circulatory fluid [11].

Dynamic viscosity of the circulatory fluid was measured at $37^{\circ} \mathrm{C}$ for a shear rate of $6000 \mathrm{~s}^{-1}$ with a rotational rheometer (Haake RheoStress 1, Thermo Fisher Scientific, USA) utilizing a cone-plate setup. The density of the circulatory fluid was determined by measuring the mass (Präzisionswaage Kern 770, Kern \& Sohn GmbH, Germany) of a known volume at $37^{\circ} \mathrm{C}$.

\subsection{Mitral valve model}

A non-calcified, pathological MVM (LifeLike BioTissue Inc., Canada) featuring a D-shaped MV annulus with an annulus area of $7.6 \mathrm{~cm}^{2}$ and an annulus circumference of $103 \mathrm{~mm}$ was used (see Figures 1A and 1B). The aorto-mural distance (Ao-M) is $30.89 \mathrm{~mm}$ and the intercommisural distance $(\mathrm{C}-\mathrm{C})$ between the antero-lateral and postero-medial commissure is $34.25 \mathrm{~mm}$. The anterior leaflet consists of a semi-circular structure with a thickness of $1.12 \mathrm{~mm}$. The posterior leaflet is made up of three scallops (P1-P3) and features a thickness of $1.11 \mathrm{~mm}$. Sufficient MV closure is ensured by two papillary muscle structures. From the tips of each of these structures, three chordae tendineae arise, inserting at the leading edges of the anterior and posterior MV leaflets. Mitral regurgitation occurs due to a flail gap in the A2-P2 region.
In order to determine the impact of the width of transcatheter MVR devices during LV-systole, three MV configurations were investigated (see Figures 1C-1E): MVM without MVR device (config. i), MVM with one MVR device (config. ii) and MVM with two MVR devices (config. iii) implanted in the A2-P2 region. The custom-made MVR devices used in this study were made from steel sheets with a thickness of $0.2 \mathrm{~mm}$ and feature an arm length of $9.0 \mathrm{~mm}$ and a width of $5.0 \mathrm{~mm}$ (see Figure 1F).

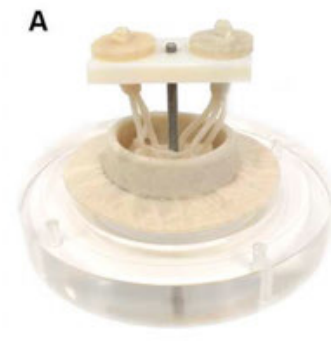

C

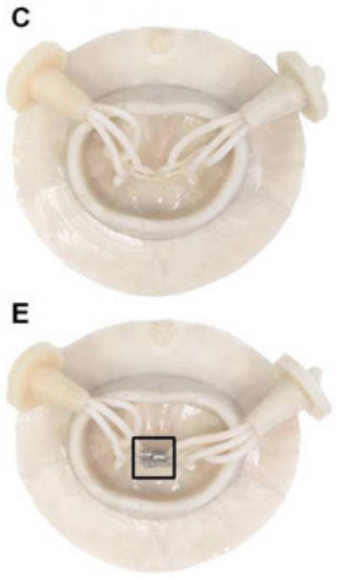

B

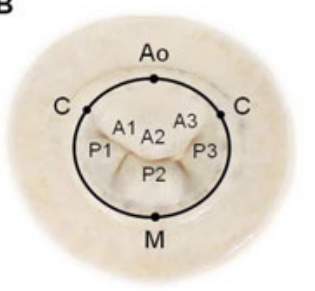

D

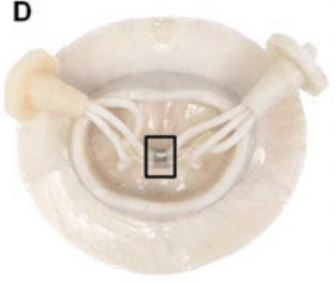

F

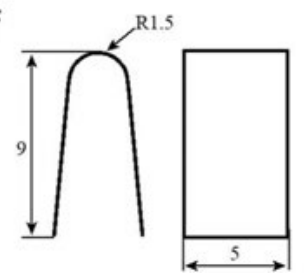

Figure 1: A) Pathological MVM with two papillary muscle structures and chordae tendineae. B) Atrial view of the MVM during LV-systole. Aorto-mural distance (Ao-M) is $30.89 \mathrm{~mm}$, the intercommisural distance (C-C) between antero-lateral and postero-medial commissure is $34.25 \mathrm{~mm}$. A1-A3 and P1-P3 indicate the segments of the anterior and the posterior leaflet, respectively. C, D, E) Ventricular view of the pathological MVM without MVR device (C), one custom-made MVR device (D) and two custom-made MVR devices (E) implanted in A2-P2 position. F) Dimensions of the custom-made MVR devices.

\section{Results and discussion}

Experimental investigations of the dynamic viscosity of the glycerol-water mixture $(36 \%(\mathrm{v} / \mathrm{v})$ glycerol in water) yield a dynamic viscosity of $3.5 \mathrm{mPa} \cdot \mathrm{s}$ at a temperature of $37^{\circ} \mathrm{C}$ and a shear rate of $6000 \mathrm{~s}^{-1}$. A density of $(1098.2 \pm 1.3) \mathrm{kg} \cdot \mathrm{m}^{-3}$ was measured for the glycerol-water mixture. 
Visual examination confirms transvalvular closing pressure gradients of $\Delta p<5 \mathrm{mmHg}$ for MVM configurations i-iii. Since the physiological LV diastolic pressure ranges from $\left(3 \leq p_{D} \leq 12\right) \mathrm{mmHg}$, the measured closing pressure gradient of $(3 \leq \Delta p \leq 7) \mathrm{mmHg}$ is within the physiological range [12].

Hydrodynamic testing of the pathological MVM without a MVR device (config. i) shows a malcoaptation manifesting in a flail gap in the A2-P2 region. The flail gap can visually be examined for transvalvular pressure gradients ranging from $(50 \leq \Delta p \leq 73) \mathrm{mmHg}$ (see Figure 2A). Image analysis yields an increasing flail gap area with increasing transvalvular pressure gradients. The maximum flail width $l_{F, \max }=1.6 \mathrm{~mm}$ along the coaptation line and the maximum distance between the free edges of $\mathrm{A} 2$ and $\mathrm{P} 2 s_{F, \max }=0.9 \mathrm{~mm}$ are measured at $\Delta p=73 \mathrm{mmHg}$. Due to the A2-P2 flail gap the highest retrograde flow $Q_{\max }=(0.79 \pm 0.01) 1 \cdot \mathrm{min}^{-1}$ for configurations i-iii was measured for config. i (see Table 1 and Figure 2D).

Moreover, the conducted investigations show that the implantation of both, one or two MVR devices, effectively prevents the manifestation of a flail gap (see Figures $2 \mathrm{~B}$ and 2C). Qualitative visual examinations of the atrial view confirm coaptation and symmetrical overlap of the anterior and posterior MV leaflets. However, the impact of the implantation of one MVR device with a width of $5 \mathrm{~mm}$ and the implantation of two MVR devices with a width of $5 \mathrm{~mm}$ each, is much more immanent in the $\Delta p-\mathrm{Q}$ relations (see Table 1 and Figure 2D).

Both, the increase of device-induced coaptation-width $(l \approx 10 \mathrm{~mm})$ and the device-induced coaptation area $\left(A \approx 90 \mathrm{~mm}^{2}\right.$ ) contribute to the increase of the maximum transvalvular pressure gradient and the decrease of the maximum volumetric flowrate during LV-systole. Thus, the increase of the investigated device-induced coaptation-width effectively prevents pathological retrograde blood flow.

Table 1: Experimentally investigated maximum transvalvular pressure gradients $(\Delta p)$ and volumetric flowrates $\left(Q_{\max }\right)$ for configurations i-iii.

\begin{tabular}{cccc}
\hline & Config. i & Config. ii & Config. iii \\
\hline$\Delta \boldsymbol{p}_{\max }$ & $73 \mathrm{mmHg}$ & $130 \mathrm{mmHg}$ & $177 \mathrm{mmHg}$ \\
$\boldsymbol{Q}_{\max }$ & $0.79 \mathrm{I} \cdot \mathrm{min}^{-1}$ & $0.69 \mathrm{I} \cdot \mathrm{min}^{-1}$ & $0.58 \mathrm{I} \cdot \mathrm{min}^{-1}$ \\
\hline
\end{tabular}
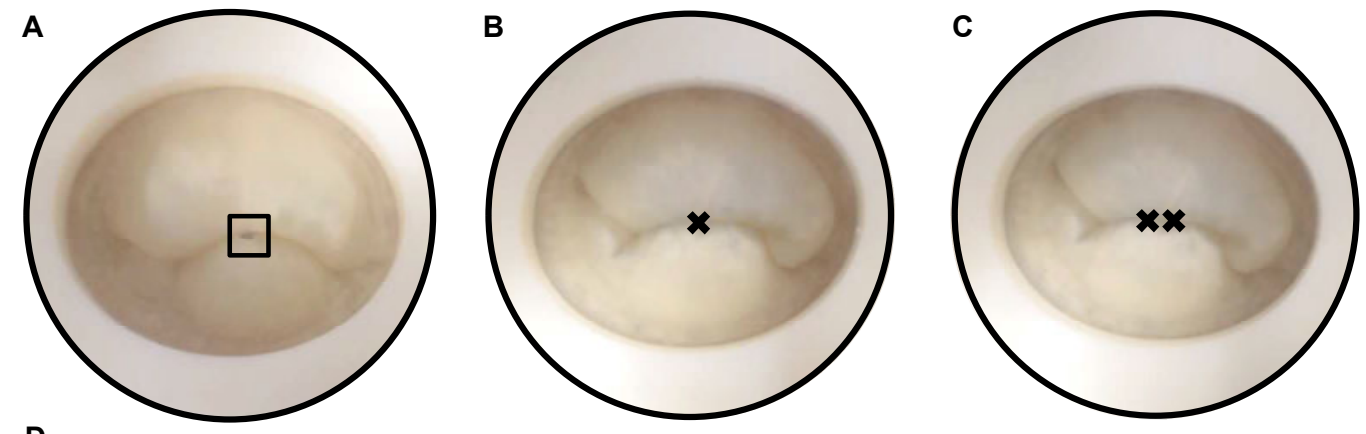

D

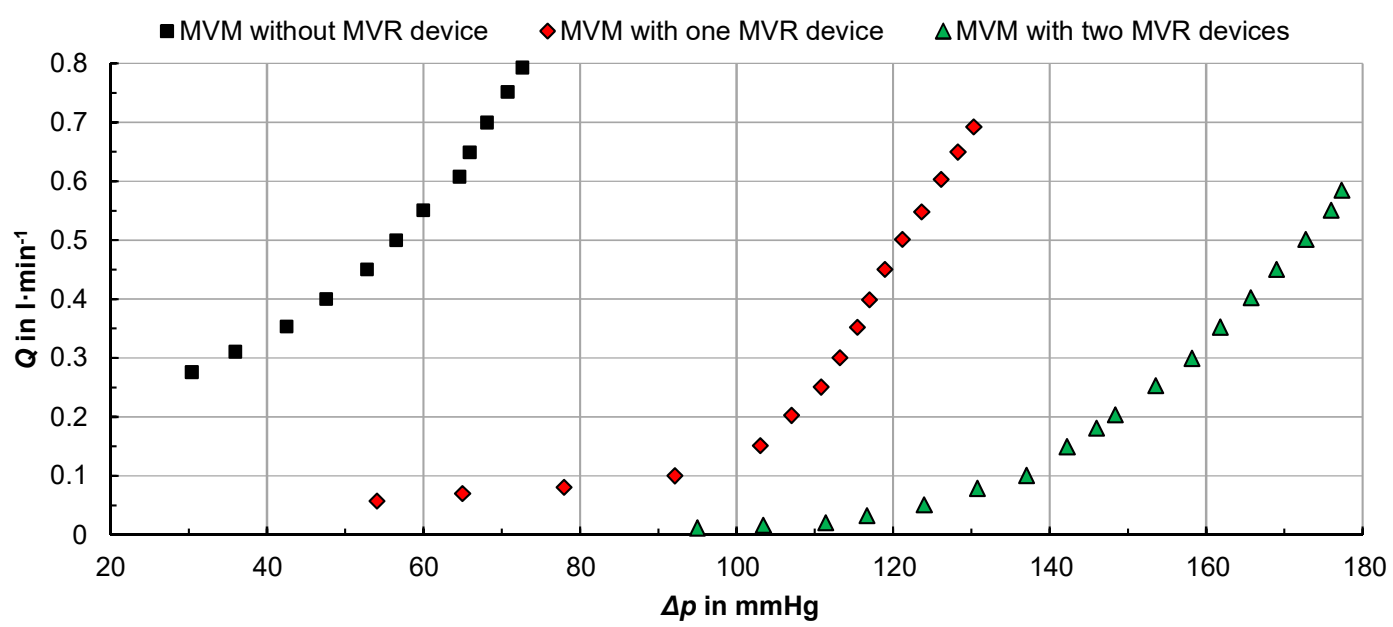

Figure 2: A-C) Atrial view of the MVM during LV-systole in the experimental setup for config. $\mathrm{i}$ at $\Delta p=73 \mathrm{mmHg}$ (A), for config. ii at $\Delta p=130 \mathrm{mmHg}(\mathrm{B})$ and config. iii at $\Delta p=177 \mathrm{mmHg}(\mathrm{C})$. The black square highlights the A2-P2 flail gap and the position of the MVR devices is marked with a black cross. D) Experimentally investigated $\Delta p-Q$ relations for configurations i-iii. 


\section{Conclusion}

Within the current study, we investigated the impact of device-induced coaptation-width and -area on the hydrodynamic performance of insufficient MVs during LV-systole.

Chordae tendineae and papillary muscles of the utilized MVM are no anatomically and physiologically correct representations but rather simplifications which allow for sufficient MV closure. However, due to the anatomical geometries, the location of the flail gap at the A2-P2 region and an MV area of $A \geq 4 \mathrm{~cm}^{2}$ the utilized MVM is applicable for the investigation of the impact of minimally invasive edgeto-edge MVR devices such as the MitralClip (Abbott, USA) and the Pascal (Edwards Lifesciences Corp., USA) system [12-14].

Moreover, the increase of the device-induced coaptation- width from $5 \mathrm{~mm}$ to $10 \mathrm{~mm}$, as employed in the Pascal system (Edwards Lifesciences Corp., USA), leads to significant improvement of the hydrodynamic MV performance [15]. Furthermore, increasing the arm length of MVR clip devices, as for example in the MitraClip XTR system (Abbott, USA), does not only result in larger deviceinduced coaptations-areas but can also extend the application range of minimally invasive edge-to-edge MVR devices to patients with more complex MV anatomies [16]. Taking everything into consideration, the conducted in vitro investigations confirm that edge-to-edge MVR devices can effectively reduce $\mathrm{MV}$ regurgitation.

In order to investigate the impact of different MVR devices on the hydrodynamic performance of the MV throughout the cardiac cycle, physiological pulsatile flow conditions will be applied in future investigations.

\section{Author Statement}

Research funding: Partial financial support by the European Regional Development Fund (ERDF) and the European Social Fund (ESF) within the collaborative research between economy and science of the state Mecklenburg-Vorpommern and by the Federal Ministry of Education and Research (BMBF) within RESPONSE "Partnership for Innovation in Implant Technology" is gratefully acknowledged. Conflict of interest: Authors state no conflict of interest. Informed consent: Informed consent is not applicable. Ethical approval: The conducted research is not related to either human or animal use.

\section{References}

[1] Di Mauro M, Gallina S, D'Amico MA et al. Functional mitral regurgitation: from normal to pathological anatomy of mitral valve. Int J Cardiol 2013;163(3) 242-248.

[2] Enriquez-Sarano M, Akins CW, Vahanian A. Mitral regurgitation. The Lancet 2009;373(9672):1382-1394.

[3] Ducas RA, White CW, Wassef AW et al. Functional mitral regurgitation: current understanding and approach to management. Can J Cardiol 2014;30(2):173-180.

[4] Wojakowski W, Baumgartner $\mathrm{H}$. The year in cardiology 2018 : valvular heart disease. Eur Heart J. 2019;00:1-10.

[5] Kodali SK, Velagapudi P, Hahn RT et al. Valvular Heart Disease in Patients $\geq 80$ Years of Age. J. Am. Coll. Cardiol. 2018;71(18):2058-2072.

[6] Bonis $\mathrm{M}$ de, Alfieri $\mathrm{O}$. The edge-to-edge technique for mitral valve repair. HSR Proc Intensive Care Cardiovasc Anesth 2010;2(1):7-17.

[7] van Praet KM, Stamm C, Sündermann SH et al. Minimally Invasive Surgical Mitral Valve Repair: State of the Art Review. Interv Cardiol 2018;13(1):14-19.

[8] Nishimura RA, Otto CM, Bonow RO et al. 2017 AHA/ACC Focused Update of the 2014 AHA/ACC Guideline for the Management of Patients With Valvular Heart Disease: A Report of the American College of Cardiology/American Heart Association Task Force on Clinical Practice Guidelines. Circulation 2017;135(25):1159-1195.

[9] Baumgartner H, Falk V, Bax JJ et al. 2017 ESC/EACTS Guidelines for the management of valvular heart disease. Eur Heart J 2017;38(36):2739-2791.

[10] Ott R, Kaule S, Grossmann S, Pfensig S, Öner A, Stiehm M, Grabow N, Schmitz KP, Siewert S. Development of an experimental setup fort he in vitro investigation of mitral valve repair devices. Curr. Dir. Biomed. Eng. (2019);5(1):501-504

[11] Lim WL, Chew YT, Chew TC et al. Particle image velocimetry in the investigation of flow past artificial heart valves. Ann Biomed Eng 1994;22(3):307-318.

[12] Klingensmith ME. The Washington manual of surgery, 5th ed. Philadelphia: Wolters Kluwer Health/Lippincott Williams \& Wilkins; 2008.

[13] Di Mauro Michele, Gallina S, D'Amico MA et al. Functional mitral regurgitation: from normal to pathological anatomy of mitral valve. Int. J. Cardiol. 2013;163(3):242-248.

[14] El Sabbagh A, Reddy YNV, Nishimura RA. Mitral Valve Regurgitation in the Contemporary Era: Insights Into Diagnosis, Management, and Future Directions. JACC: Cardiovasc. imaging 2018;11(4):628-643.

[15] Praz F, Spargias K, Chrissoheris M et al. Compassionate use of the PASCAL transcatheter mitral valve repair system for patients with severe mitral regurgitation: a multicentre, prospective, observational, first-in-man study. The Lancet 2017;390(10096):773-780.

[16] Jaworek M, Gelpi G, Romagnoni C et al. (2019): Long-arm Clip for Transcatheter Edge-to-Edge Treatment of Mitral and Tricuspid Regurgitation - Ex-Vivo Beating Heart Study. Structural Heart 2019;3(3): 211-219. 